\section{An analysis of a polymer additive for calcium carbonate crystallization - a small-angle neutron scattering investigation by applying contrast variation}

\author{
Hitoshi Endo, ${ }^{\text {ab }}{ }_{*}$ Helmut Cölfen ${ }^{\mathrm{a}}$ and Dietmar Schwahn ${ }^{\mathrm{b}}$ \\ ${ }^{a}$ Max Planck Institute of Colloids and Interfaces, Colloid Chemistry, Research \\ Campus Golm, D-14424 Potsdam, Germany, ${ }^{b}$ Institut für Festköperforschung, \\ Forschungszentrum Jülich, D-52425 Jülich, Germany. E-mail: h.endo@fz-juelich.de
}

The role of the double-hydrophilic block copolymers (poly(ethylene glycol)-block-poly(methacrylic acid)) on the morphogenesis of calcium carbonate $\left(\mathrm{CaCO}_{3}\right)$ was studied by applying contrast variation small-angle neutron scattering (SANS). The scattering function for ternary systems can be expressed by only partial scattering functions of the single species, as derived on the assumption of incompressibility. We demonstrate the feasibility of this formula by means of contrast variation SANS, and the decomposed partial scattering functions give their detailed information. Especially the partial scattering function of polymer shows a $Q^{-2.7}$ monotonic slope at low $Q(0.002 \AA$ $<Q<0.02 \AA ; Q$ is the modulus of the scattering vector), which is a clear evidence that the distribution of the polymer in $\mathrm{CaCO}_{3}$ has a mass fractal dimension. This result is confirmed by time-resolved SANS experiments with $\mathrm{CaCO}_{3}$ contrast, where a $Q^{-2.5}$ slope was observed at the early stage of the reaction in the same $Q$ range.

Keywords: contrast variation; neutron scattering; calcium carbonate.

\section{Introduction}

The morphology-controlled synthesis of inorganic materials is a key aspect not only in the field of material science, as ceramics, catalysis, pigments, cosmetics, and so on (Mann \& Ozin, 1996). It is also well known that biological systems synthesize highly advanced and optimized inorganic materials such as bones, teeth, and shells by controlling their size, shape, structure and orientation with the help of selfassembled organic molecules like proteins, lipids, etc. In this article we investigated calcium carbonate $\left(\mathrm{CaCO}_{3}\right)$ crystals which are influenced by double-hydrophilic block copolymer poly(ethylene glycol)-blockpoly(methacrylic acid) (PEG- $b$-PMAA) with respect to their morphology and size. In our system, these $\mathrm{CaCO}_{3}$ crystals are synthesized by room temperature crystallization from water in the presence of PEG- $b$ PMAA, and it has been found that the morphology is mainly affected by the ratio [polymer] $/\left[\mathrm{CaCO}_{3}\right]$ and the $\mathrm{pH}$ of the solution (Cölfen \& Qi, 2001).

The aim of this manuscript is to investigate the role of double hydrophilic block copolymers for the formation of $\mathrm{CaCO}_{3}$ particle superstructures by using contrast variation small-angle neutron scattering (SANS). Since the scattering signal from polymer was very weak, we needed very careful contrast variation experiments as well as theoretical developments to evaluate the data quantitatively. We proceed further derivation of the scattering theory on the basis of partial scattering functions and demonstrate its experimental feasibility.

Furthermore, we studied the kinetics of the reaction by means of time-resolved SANS measurements, where a gas-liquid diffusion reaction was used to realize a slow reaction speed. We investigated the effect of the polymer concentration on the reaction through these experiments.

\section{2. $\mathrm{CaCO}_{3}$ synthesis}

We prepared $\mathrm{CaCO}_{3}$ crystals based on the knowledge given by Cölfen \& Qi (2001). A double hydrophilic block copolymer PEG- $b$-PMAA $(\mathrm{PEG}=3000 \mathrm{~g} / \mathrm{mol}$, PMAA $=700 \mathrm{~g} / \mathrm{mol})$, which was obtained from Goldschmit AG, Essen (Germany), was used as a template polymer, where the PMAA block interacts strongly with inorganic material while the PEG block mainly promotes the solubility in water (Cölfen, 2001). All other chemicals were purchased from Aldrich and used without further purification.

The synthesis of $\mathrm{CaCO}_{3}$ was carried out by means of precipitation, i.e., an aqueous solution of $\mathrm{Na}_{2} \mathrm{CO}_{3}$ and PEG- $b$-PMAA was first prepared and adjusted to a desired $\mathrm{pH}$ (e.g., $\mathrm{pH}=10$ ) by using $\mathrm{HCl}$ or $\mathrm{NaOH}$, then a solution of $\mathrm{CaCl}_{2}$ was mixed quickly with the $\mathrm{pH}$ adjusted solution under vigorous stirring at room temperature. A detailed description is given by Cölfen \& Qi (2001).

The ion concentrations both of $\mathrm{CaCl}_{2}$ and $\mathrm{Na}_{2} \mathrm{CO}_{3}$ were 0.4 normal $(N)$, which is much higher than in the case of Cölfen \& Qi (2001), and a polymer concentration was prepared with a mass ratio [polymer $] /\left[\mathrm{CaCO}_{3}\right]=0.9$. After the reaction the crystals were separated from the polymer solution by sedimentation and rinsed three times with pure water, so that the polymer which did not react with $\mathrm{CaCO}_{3}$ was removed almost completely. The obtained crystals were checked by optical microscopy, and we could confirm the expected morphology was obtained. Fig. 1 depicts the electron microscopy pictures of the obtained materials. The effect of polymer is clearly shown, that is, without polymer only rhombohedral crystals (calcite) are obtained, on the other hand, mainly a dumbbell structure is obtained with polymer.

\section{Small-angle neutron scattering}

SANS experiments were performed at KWS-1 SANS diffractometer at the FRJ-2 reactor of the Forschungszentrum Jülich ${ }^{1}$. We used a neutron wavelength $\lambda=7 \AA$ with $\Delta \lambda / \lambda=20 \%$ full width at half-maximum (FWHM). The covered $Q$ range was $0.002 \AA^{-1}<Q<0.2 \AA^{-1}$ in reciprocal space, where $Q$ is the modulus of the scattering vector defined by $Q=4 \pi \sin (\theta / 2) / \lambda$ with the scattering angle $\theta$.

The calcium carbonate $\left(\mathrm{CaCO}_{3}\right)$ crystals were mixed with $\mathrm{D}_{2} \mathrm{O}$ and $\mathrm{H}_{2} \mathrm{O}$ mixture for performing the contrast variation experiments. To avoid the sedimentation of the crystals, a special type of cell was prepared which has both an input- and an output-nozzle at the top and bottom. A silicon tube was connected between the cell and a peristaltic pump circulated the solution during the experiments.

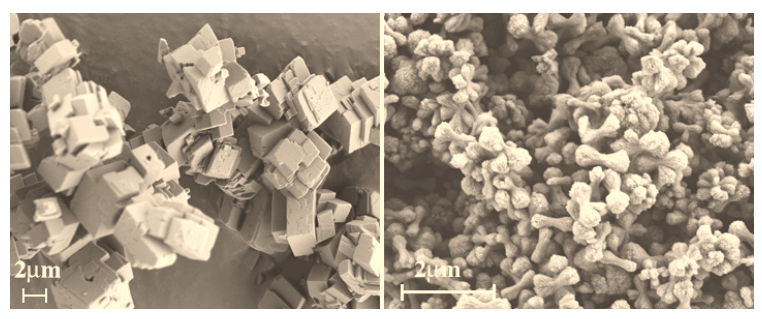

Figure 1

Transmission electron micrographs for $\mathrm{CaCO}_{3}$ particles with different morphologies: prepared without polymer (left) and with polymer (right).

The data sets were normalized to absolute intensity by the incoherent scattering of a Lupolen secondary standard.

\footnotetext{
${ }^{1}$ The instrumental details of KWS-1 are given on http://www.fz-juelich.de/iff/Institute/ins/Broschuere_NSE/kws1.shtml
} 


\section{Contrast variation}

\subsection{Scattering theory}

It is well known that the elastic coherent scattering intensity $I(Q)$ for a multicomponent system with $p$ different species is given by

$$
I(Q)=\sum_{i=1}^{p} \rho_{i}^{2} S_{i i}(Q)+2 \sum_{i<j}^{p} \rho_{i} \rho_{j} S_{i j}(Q)
$$

where $S_{i j}(Q)$ is a partial scattering function defined by

$$
S_{i j}(Q)=\frac{1}{V} \int_{V^{\prime}} \int_{V}\left\langle\phi_{i}(\vec{r}) \phi_{j}\left(\overrightarrow{r^{\prime}}\right)\right\rangle \exp \left\{i \vec{Q} \cdot\left(\vec{r}-\vec{r}^{\prime}\right)\right\} \mathrm{d}^{3} \vec{r} \mathrm{~d}^{3} \overrightarrow{r^{\prime}}
$$

with the local volume fraction of component $i, \phi_{i}(\vec{r})$ (see $e . g$., Bacon, 1975; or Higgins \& Benoit, 1994). In the case of neutron scattering, $\rho_{i}$ is the scattering length density of component $i$ defined by

$$
\rho_{i}=\frac{\sum_{z} b_{z}}{v_{i}}
$$

where $b_{z}$ is the coherent scattering length of the atoms in component $i$ and $v_{i}$ is the corresponding volume.

Benoit et al. (1981) demonstrated a useful expression based on the assumption of incompressibility (or Babinet's principle):

$$
I(Q)=\sum_{i \neq k}^{p}\left(\rho_{i}-\rho_{k}\right)^{2} S_{i i}(Q)+2 \sum_{i, j \neq k ; i<j}^{p}\left(\rho_{i}-\rho_{k}\right)\left(\rho_{j}-\rho_{k}\right) S_{i j}(Q),
$$

where all the partial scattering functions involving component $k$ are canceled, since the condition of incompressibility gives the relationship for partial scattering functions as

$$
\sum_{i=1}^{p} S_{i k}(Q)=0
$$

where component $k$ can be arbitrarily chosen (Higgins \& Benoit, 1994).

For ternary system with species A, B and C, equation (4) leads to

$$
\begin{array}{r}
I(Q)=\left(\rho_{\mathrm{A}}-\rho_{\mathrm{C}}\right)^{2} S_{\mathrm{AA}}(Q)+2\left(\rho_{\mathrm{A}}-\rho_{\mathrm{C}}\right)\left(\rho_{\mathrm{B}}-\rho_{\mathrm{C}}\right) S_{\mathrm{AB}}(Q) \\
+\left(\rho_{\mathrm{B}}-\rho_{\mathrm{C}}\right)^{2} S_{\mathrm{BB}}(Q) .
\end{array}
$$

On the other hand, equation (5) leads to

$$
\begin{array}{r}
2 S_{\mathrm{AB}}(Q)=-S_{\mathrm{AA}}(Q)-S_{\mathrm{BB}}(Q)+S_{\mathrm{CC}}(Q) \\
2 S_{\mathrm{BC}}(Q)=S_{\mathrm{AA}}(Q)-S_{\mathrm{BB}}(Q)-S_{\mathrm{CC}}(Q) \\
2 S_{\mathrm{CA}}(Q)=-S_{\mathrm{AA}}(Q)+S_{\mathrm{BB}}(Q)-S_{\mathrm{CC}}(Q)
\end{array}
$$

All the cross terms of partial scattering functions in equation (1) can be replaced with squared terms by substituting equation (7), and we achieve

$$
\begin{aligned}
I(Q) & =\left(\rho_{\mathrm{A}}-\rho_{\mathrm{B}}\right)\left(\rho_{\mathrm{A}}-\rho_{\mathrm{C}}\right) S_{\mathrm{AA}}(Q) \\
& +\left(\rho_{\mathrm{B}}-\rho_{\mathrm{A}}\right)\left(\rho_{\mathrm{B}}-\rho_{\mathrm{C}}\right) S_{\mathrm{BB}}(Q) \\
& +\left(\rho_{\mathrm{C}}-\rho_{\mathrm{A}}\right)\left(\rho_{\mathrm{C}}-\rho_{\mathrm{B}}\right) S_{\mathrm{CC}}(Q)
\end{aligned}
$$

for any ternary system. Equations (6) and (8) are based on a completely equal level of approximation.

\subsection{Contrast variation SANS experiment}

In this study the three components are $\mathrm{CaCO}_{3}$, polymer, and water, so that if the contrast between $\mathrm{CaCO}_{3}$ and water is completely matched, the scattering signal should come only from the polymer, e.g., if $\rho_{\mathrm{A}}=\rho_{\mathrm{B}}$ in equation (8), the scattering intensity $I(Q)$ arises from only $S_{\mathrm{CC}}(Q)$. However, if the concentration of the polymer in $\mathrm{CaCO}_{3}$ is very small, the contrast mismatch between $\mathrm{CaCO}_{3}$ and water may seriously disturb the signal from polymer, even though the mismatching is very small.

To avoid this problem, we performed contrast variation experiments. As a starting point, a $\mathrm{D}_{2} \mathrm{O} / \mathrm{H}_{2} \mathrm{O}$ mixture was prepared to match the scattering length density $4.90 \times 10^{10} \mathrm{~cm}^{-2}$, which is slightly larger than that of $\mathrm{CaCO}_{3}\left(4.69 \times 10^{10} \mathrm{~cm}^{-2}\right)$. A stepwise reduction of the scattering length density of water was achieved by adding minute amounts of $\mathrm{H}_{2} \mathrm{O}$, which is between 20 and $60 \mathrm{mg}$ corresponding to approximately 1-3 wt.\% of the total amount of solvent. The addition of $\mathrm{H}_{2} \mathrm{O}$ was done with a microsyringe and the mass of additional $\mathrm{H}_{2} \mathrm{O}$ was checked with a chemical balance. Five measurements with different water contrast were performed, which covers between $4.48 \times 10^{10}$ and $4.90 \times 10^{10} \mathrm{~cm}^{-2}$ of the scattering length density.

In Fig. 2 the obtained scattering profiles of the contrast variation experiments are shown. It is clearly shown that the intensity at low $Q$ first decreases with the addition of $\mathrm{H}_{2} \mathrm{O}$ (Fig. 2a), and increases after the scattering contrast of the aqueous solution crosses the value of $\mathrm{CaCO}_{3}$ (Fig. 2b). The data sets in Fig. 2 and an additional " $\mathrm{D}_{2} \mathrm{O}$ contrast" result, which was obtained by using $\mathrm{D}_{2} \mathrm{O}$ only as the aqueous solution, were decomposed into partial scattering function. The " $\mathrm{D}_{2} \mathrm{O}$ contrast" result improved the stability of the computation. Equation (8) contains three unknowns, i.e., $S_{\mathrm{AA}}(Q), S_{\mathrm{BB}}(Q)$, and $S_{\mathrm{CC}}(Q)$; therefore the use of the full experimental data set (five contrast variations plus " $\mathrm{D}_{2} \mathrm{O}$ contrast") leads to an overdetermined set of 6 equations. These equations were solved by singular-value decomposition for each $Q$ value on the basis of Equation (8) under the assumtion that all the scattering contrasts are known from the sample preparation.

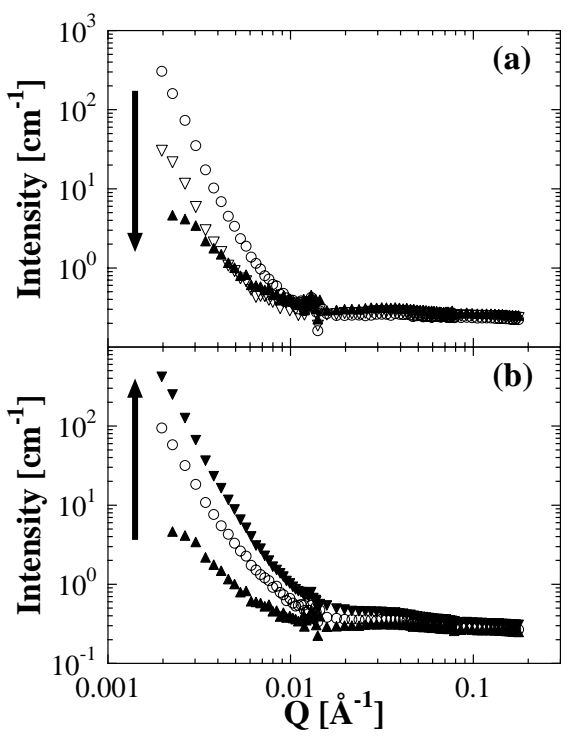

Figure 2

Contrast variation series from $\mathrm{CaCO}_{3}$ prepared with the polymer: $(a)$ increment I ( $(\circ)$, II $(\nabla)$, and III $(\boldsymbol{\Lambda}) ;(b)$ increment III $(\boldsymbol{\Lambda})$, IV $(\circ)$, and V $(\boldsymbol{\nabla})$. The calculated scattering length densities of water for each addition are I 4.896, II 4.811, III 4.730, IV 4.642 , and V $4.476\left[\times 10^{10} \mathrm{~cm}^{-2}\right]$, respectively. 


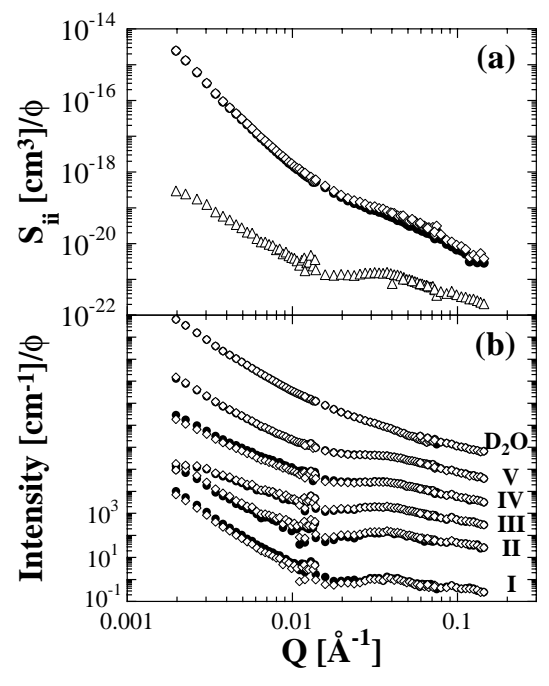

Figure 3

(a) Decomposed partial scattering functions of $\mathrm{CaCO}_{3} S_{\mathrm{cc}}(\diamond)$, water $S_{\mathrm{ww}}(\bullet)$ and polymer $S_{\mathrm{pp}}(\triangle)$ respectively, normalized by volume fraction. (b) Comparison between the obtained scattering intensities $(\bullet)$ and the reconstracted intensities $(\diamond)$. The curves are separated from each other by a shift factor of 10 or 100. The intensity scale applies directly to the lowermost curve. The numbers beside the curves indicate the corresponding increments shown in Fig. 2.

Fig. 3(a) shows the obtained three partial scattering functions of water, $S_{\mathrm{ww}}(Q) ; \mathrm{CaCO}_{3}, S_{\mathrm{cc}}(Q)$; and polymer, $S_{\mathrm{pp}}(Q)$. The scattering intensities were reconstructed on the basis of the obtained partial scattering functions, i.e., the back-substitution of the partial scattering functions in equation (8) leads to the corresponding scattering intensities. The reconstructed intensities are compared with the experimental results in Fig. 3(b). A nearly perfect reconstruction is achieved. This result confirms that the model given by equation (8) and the calculated scattering length densities are appropriate.

\section{Analysis of partial scattering functions}

\section{1. $S_{\mathrm{ww}}$ and $S_{\mathrm{cc}}$}

Fig. 4(a) displays the resulting partial scattering functions of water $\left(S_{\mathrm{ww}}\right)$ and $\mathrm{CaCO}_{3}\left(S_{\mathrm{cc}}\right)$. These obtained partial scattering functions are quite resonable from the point of view explained below: first of all, each resulting partial scattering function is positive. That is the demand of the squared terms of partial scattering functions. Secondly, $S_{\mathrm{cc}} \approx S_{\mathrm{ww}}$ is fulfilled for whole $Q$ range, which is a clear evidence that the polymer concentration in the $\mathrm{CaCO}_{3}$ crystals is very small, therefore the system can be regarded as pseudo-binary.

$S_{\mathrm{ww}}$ and $S_{\mathrm{cc}}$ consist of two parts: at low $Q$, a $Q^{-4}$ slope is clearly shown, which corresponds to the interface scattering (Porod's law), and at high $Q$ an additional scattering is observed. This part was fitted by the Guinier function and an additional fluctuation term based on Beaucage's approach (Beaucage, 1995), that is

$$
I_{\text {high } Q}(Q) \propto\left\{\exp \left(\frac{-Q^{2} R_{\mathrm{g}}^{2}}{3}\right)+2 \pi \frac{S}{V}\left[\frac{\left\{\operatorname{erf}\left(Q R_{\mathrm{g}} / \sqrt{6}\right)\right\}^{3}}{Q}\right]^{4}\right\}
$$

where $S / V$ is the specific amount of surface. A radius of gyration of $R_{\mathrm{g}}=41 \AA$ is obtained. We assume that small particles are attached to sharp interface.

\subsection{The polymer partial scattering function $S_{\mathrm{pp}}$}

The extracted partial scattering function of polymer $S_{\mathrm{pp}}(Q)$ displayed in Fig. 4(b) contains information about the used doublehydrophilic block copolymer PEG- $b$-PMAA in the $\mathrm{CaCO}_{3}$ crystals. $S_{\mathrm{pp}}(Q)$ may be composed of two parts, namely, on one hand polymercoil scattering is observed at high $Q$; on the other hand, the intensity increases monotonically at low $Q$. This monotonic slope at low $Q$, which shows nearly $I(Q) \propto Q^{-3}$, could be a clear evidence of a large structure formed by the polymer in $\mathrm{CaCO}_{3}$. Moreover, we assume that this slope proportional to $Q^{-3}$ originates from a mass fractal dimension. To evaluate this scattering profile, we applied the function

$$
I_{\mathrm{M}}(Q) \propto-\cos \left(\frac{D_{\mathrm{f}} \pi}{2}\right) \Gamma\left(D_{\mathrm{f}}-1\right) Q^{-D_{\mathrm{f}}}
$$

where $\Gamma(x)$ is the gamma function and $D_{\mathrm{f}}$ is the mass fractal dimension with $2<D_{\mathrm{f}}<3$. The corresponding correlation function $G(r)$ is given by

$$
G(r) \propto r^{D_{\mathrm{f}}-D}
$$

with the space dimension $D$, and $D=3$ in this case (Sinha et al., 1984; Freltoft et al., 1986). At small $r$ in equation (11), the polymer centers cannot approach closer than a radius $2 R_{\mathrm{g}}$, so that $G(r)=0$ for $r<2 R_{\mathrm{g}}$. In this situation, equation (10) decreases quickly and the scattering intensity is dominated by the polymer-coil scattering, which is described as

$$
I_{\text {coil }}(Q) \propto\left\{\exp \left(\frac{-Q^{2} R_{\mathrm{g}}^{2}}{3}\right)+B\left[\frac{\left\{\operatorname{erf}\left(w Q R_{\mathrm{g}} / \sqrt{6}\right)\right\}^{3}}{Q}\right]^{P}\right\}
$$

with $Q^{-P}$ asymptote with the Flory exponent $P=5 / 3$ for a swollen polymer chain, the radius of gyration of polymer $R_{\mathrm{g}}$, and $w=1.06$ (Beaucage, 1996). $B$ is a prefactor and defined by

$$
B=\left(P / R_{\mathrm{g}}^{P}\right) \Gamma(P / 2)
$$

Equation (12) is close to the Debye equation for an ideal (Gaussian) chain. Therefore we fitted the data with equation (10) at low $Q$ $\left(Q<0.01 \AA^{-1}\right)$ and with equation (12) at high $Q\left(Q>0.02 \AA^{-1}\right)$.

In Fig. $4(b)$ the obtained partial scattering functon $S_{\mathrm{pp}}(Q)$ and the corresponding fitting curves with equations (10) and (12) are shown. The obtained main parameters are $R_{\mathrm{g}}=32.6 \AA$, which is quite reasonable for its molecular weight, and $D_{\mathrm{f}}=2.72$. 


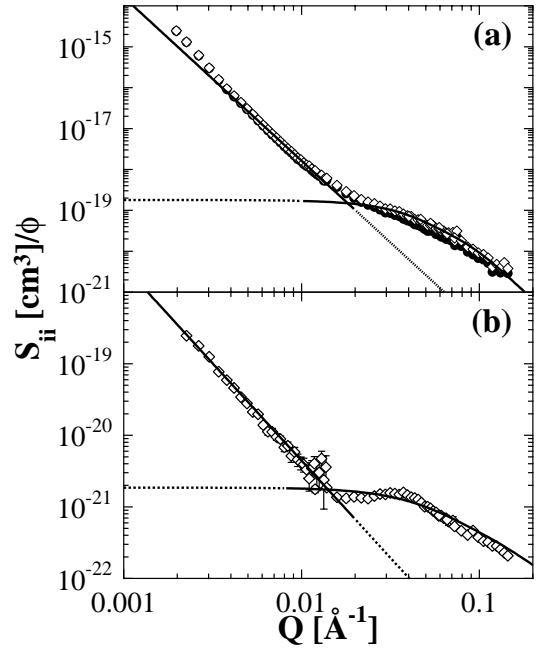

Figure 4

Partial scattering functions with fitting curves. (a) $S_{\mathrm{cc}}(\diamond)$ and $S_{\mathrm{ww}}(\bullet)$. Lines correspond to fits with $I \propto Q^{-4}$ at low $Q$ and Beaucage's approach at high $Q$. (b) $S_{\mathrm{pp}}(\diamond)$ with fitting curves by equation (10) at low $Q$ and equation (12) at high $Q$. See the text.

We believe that the fractal dimension $D_{\mathrm{f}} \approx 2.7$ means the distribution of polymer in $\mathrm{CaCO}_{3}$ crystals shows mass fractal, which should be like a dendritic structure or a percolationg network, over a large scale. Such structures with $D_{\mathrm{f}} \approx 2.5$ can be observed commonly for aggregation behavior like silica particle aggregates, silica aerogels, polymer gels, etc. Furthermore $D_{\mathrm{f}} \approx 2.5$ can be observed often for non-equlibrium growth processes like diffusion limited aggregations or percolation clusters (Schaefer et al., 1985). So our result suggests that the structure formation of $\mathrm{CaCO}_{3}$ with the double hydrophilic block copolymer can be classified to these phenomena.

\section{Time-resolved SANS measurement}

Time-resolved SANS experiments were performed in order to study nucleation kinetics of calcium carbonate $\left(\mathrm{CaCO}_{3}\right)$ in the presence of double hydrophilic block copolymer (DHBC). To achieve a slow $\mathrm{CaCO}_{3}$ crystallization, gas phase diffusion reaction was applied, i.e., ammonium-carbonate and aqueous solution with the calcium ions and DHBC put together in a special chamber equipped with a thermostat, and which is sealed from outer atmosphere. Ammonium-carbonate generates carbon dioxide gas in the chamber, and which very slowly reacts inside the aqueous solution (Yu et al., 2001). The obtained $\mathrm{CaCO}_{3}$ particles were checked by optic microscopy, and the morphology for the time period of SANS experiments was identical to that of Cölfen \& Qi (2001).

In Fig. 5 the obtained SANS curves with $\mathrm{CaCO}_{3}$ contrast (the aqueous solution is $\mathrm{H}_{2} \mathrm{O}$ ) are shown. The sample was prepared with $0.1 \mathrm{M}$ $\mathrm{CaCl}_{2}$ and 3 wt. $\%$ DHBC at $303 \mathrm{~K}\left(30^{\circ} \mathrm{C}\right)$ and $\mathrm{pH}=7 . Q^{-D}$ behavior was observed at the given reciprocal $Q$-range for $15 \mathrm{~min}$ timeresolution, which the SANS instrument at the research reactor in Jülich typically has for these samples at $20 \mathrm{~m}$ detector distance.

The curves were fitted with

$$
I(Q)=A Q^{-D}+I_{\text {incoh }}
$$

where $A$ is a prefactor and $I_{\text {incoh }}$ is a incoherent scattering intensity, which is $Q$-independent (constant). The obtained results of the time evoluton of the index $D$ in equation (14) are summed up in Fig. 6(a), where the results of other DHBC concentrations are displayed together. At the lower DHBC concentration, the index $D$ immediately reaches nearly 4 which is interfacial scattering (Porod's low); on the other hand, at $3 \mathrm{wt} \%$ of the polymer concentration, a gradual time-evolution of $D$ from 2.5 to 4 is observed. This tendency also supports the observation of the partial scattering function of polymer $S_{\mathrm{pp}}$ described in section 5.2., that the addition of DHBC induces aggregation-like crystallization.

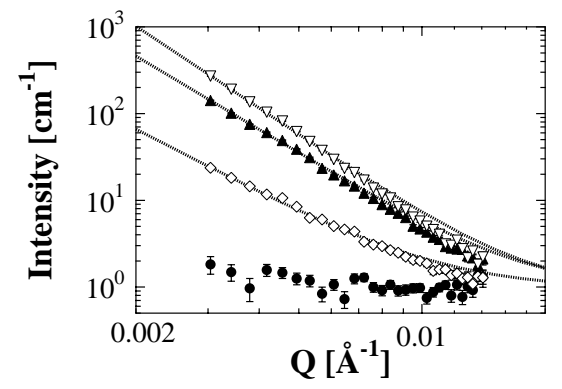

Figure 5

Time-resolved SANS profiles at $20 \mathrm{~m}$ detector distance with $0.1 \mathrm{M} \mathrm{Ca}^{2+}$ and $3 \mathrm{wt} . \%$ polymer concentration: after $0 \mathrm{~min}(\bullet), 60 \mathrm{~min}(\diamond), 120 \mathrm{~min}(\boldsymbol{\Delta})$, and $180 \mathrm{~min}(\nabla)$, respectively. The corresponding fitting using equation (14) yielded $D=2.5$ for $60 \mathrm{~min}, D=2.8$ for $120 \mathrm{~min}$, and $D=3.2$ for $180 \mathrm{~min}$, respectively.

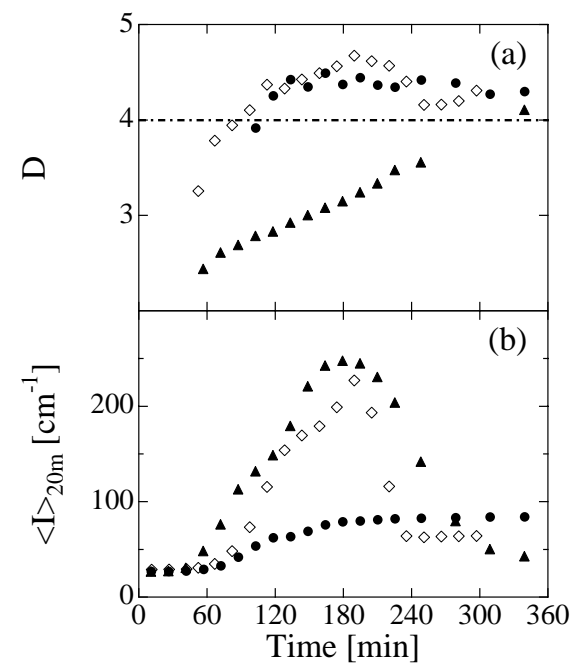

Figure 6

Effect of the polymer concentration on time evolution of (a) the index $D$ in equation (14) and $(b)$ the mean intensity at $20 \mathrm{~m}$ detector distance: $0.3 \mathrm{wt} . \%$

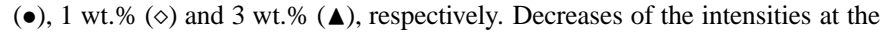
late reaction stage correspond to precipitation of $\mathrm{CaCO}_{3}$ particles.

Fig. 6(b) shows the mean intensity from the finite $Q$-range at $20 \mathrm{~m}$ detector distance. It is clear that higher concentration leads to a more rapid increase of intensity, and this is also a clear evidence that the addition of the polymer strongly affects the reaction process. ${ }^{2}$

\footnotetext{
$\overline{2}$ This quantity should be related with the prefactor $A$ in equation (14), then we are able to discuss the meaning quantitatively. However, the index $D$ changes from 2.5 to 4 continuously, which is a transition from mass fractal to surface fractal, therefore the detailed evaluation of the prefactor $A$ needs more discussions (see Endo et al., 2003).
} 


\section{conference papers}

\section{Conclusions}

We have studied the effect of the double-hydrophilic block copolymer (DHBC) PEG- $b$-PMAA on the morphogenesis of calcium carbonate $\left(\mathrm{CaCO}_{3}\right)$. It has been demonstrated that DHBC controls the synthesis of $\mathrm{CaCO}_{3}$, which has been reported in detail by Cölfen \& Qi (2001).

Small-angle neutron scattering experiments were performed applying a sophisticated contrast variation scheme, which leads to decomposition of the obtained scattering data into the partial scattering functions of each component. This is an essential requirement for a comparison with quantitative approaches and for a detailed analysis of the role of the polymer on the superstructure formation of $\mathrm{CaCO}_{3}$. Furthermore, our experiments confirm the validity of the model based on the assumption of incompressibility as well as the Babinet principle, which predicts that the scattering intensities of ternary system can be described by the three squared-terms of partial scattering functions.

The performed careful analysis of the partial scattering functions elucidates very detailed information on each structure on the mesoscopic scale. The obtained partial scattering function of polymer shows monotonic increase obeying $I(Q) \propto Q^{-2.7}$ at low $Q$, and this gives a clear evidence that the polymer distributes in $\mathrm{CaCO}_{3}$ with a mass fractal dimension. The slope starts from $Q \approx 0.002 \AA^{-1}$, which is the lowest limit of our measurement (the corresponding length scale is $2 \pi / Q \approx 300 \mathrm{~nm}$ ), so that this structure exists over a large scale (possibly up to near micrometers).

This observation is strongly supported by time-resolved SANS experiments performed under $\mathrm{CaCO}_{3}$ contrast for the investigation of kinetics of $\mathrm{CaCO}_{3}$ crystallization, where $I(Q) \propto Q^{-2.5}$ is clearly observed at the early stage of the reaction when the polymer concentration is sufficiently high.
We confirm that our experiments applying contrast variation neutron scattering is sufficiently accurate to reveal the hidden information even though the amount of the target material in the system is minute. This technique can be easily applied to other systems to achieve the information of structure on the nano- to mesoscale.

The authors acknowledge the financial support by Bundesministerium für Bildung und Forschung under Grant No. COE3MP and by the Max Planck Society. One of us (H.E.) thanks Professor D. Richter, M. Monkenbusch and W. Pyckhout for helpful discussions.

\section{References}

Bacon, G. E. (1975). Neutron Diffraction, p. 561. Oxford: Clarendon Press.

Beaucage, G. (1995). J. Appl. Cryst. 28, 717-728.

Beaucage, G. (1996). J. Appl. Cryst. 29, 134-146.

Benoit, H., Koberatein, J. \& Leibler, L. (1981). Makromol. Chem. Suppl. 4, 8599.

Cölfen, H. (2001). Macromol. Rapid Commun. 22, 219-252.

Cölfen, H. \& Qi, L. (2001). Chem. Eur. J. 7, 106-116.

Endo, H., Schwahn, D. \& Cölfen, H. (2003). In preparation.

Freltoft, T., Kjems, J. \& Sinha, S. K. (1986). Phys. Rev. B, 33, 269-275.

Higgins, J. S. \& Benoit, H. (1994). Polymers and Neutron Scattering, section 5. Oxford: Clarendon Press.

Mann, S. \& Ozin, G. A. (1996). Nature, 382, 313-318.

Schaefer, D. W., Martin J. E. \& Keefer K. D. (1985). Physics of Finely Divided Matter, edited by N. Bocarra \& M. Daoud, p. 31. Berlin: Springer-Verlag.

Sinha, S. K., Freltoft, T. \& Kjems, J. (1984). Kinetics of Aggregation and Gelation, edited by F. Family \& D. Landau, pp. 87-90. Amsterdam: NorthHolland.

Yu, S. -H., Cölfen, H. \& Antonietti, M. (2002). Adv. Funct. Mater. 12, 541-545. 